\title{
Prótese de íris, na aniridia traumática, como tentativa de controlar glaucoma refratário provocado pela presença de óleo de silicone na câmara anterior: relato de caso
}

\author{
Iris prosthesis in traumatic aniridia as an attempt to control refractory glaucoma \\ induced by silicone oil in the anterior chamber:case report
}

\author{
Rogil José de Almeida Torres ${ }^{1}$ \\ Andréa Luchini² \\ Rog'ério João de Almeida Torres ${ }^{3}$ \\ Fernando César Abib ${ }^{4}$ \\ Reginaldo Antônio de Almeida Torres ${ }^{5}$
}

\begin{tabular}{|l|}
\hline RESUMO \\
\hline O objetivo deste trabalho é demonstrar a eficácia da prótese iriana na \\
resolução do glaucoma refratário, provocado pela presença de óleo de \\
silicone na câmara anterior. Trata-se de paciente que sofreu trauma por \\
estilhaços de projétil de arma de fogo. A cirurgia vítreo-retiniana visou a \\
remoção dos corpos estranhos intra-oculares e posicionamento da retina, \\
que se encontrava descolada. Devido à ausência parcial do tecido iriano \\
e a afacia, o óleo de silicone introduzido na câmara vítrea, para manter a \\
retina colada, migrou para a câmara anterior e provocou gradativa diminui- \\
ção do número de células endoteliais e aumento da pressão intra-ocular \\
incontrolável clinicamente. Optamos pela fixação transescleral da prótese \\
de íris para corrigir tais complicações. Após 45 meses de evolução, a \\
acuidade visual estabilizou-se em conta dedos a 1 metro e a pressão intra- \\
ocular em 14 mmHg. Concluímos que a tríade composta pela ausência do \\
diafragma iriano, afacia e impossibilidade da remoção do óleo de silicone, \\
devido a inexorável recorrência de descolamento de retina, deve levar o \\
cirurgião a ponderar sobre a fixação transescleral da prótese de íris. Esta \\
conduta poderá controlar a pressão intra-ocular e/ou preservar a transpa- \\
rência corneana, impedindo o contato do óleo de silicone com a malha \\
trabecular e com o endotélio corneano.
\end{tabular}

Descritores: Óleos de silicone/efeitos adversos; Aniridia; Doenças da íris/cirurgia; Câmara anterior/cirurgia; Implante de próteses; Glaucoma; Relato de caso

Trabalho realizado no Centro Oftalmológico de Curitiba.

${ }^{1}$ Aluno do MBA - Instituto da Visão da Universidade Federal de São Paulo (UNIFESP). São Paulo (SP)

Médico do Centro Oftalmológico de Curitiba. Curitiba (PR).

${ }^{3}$ Mestre em Cirurgia pela Faculdade Evangélica do Paraná. Curitiba (PR).

Doutor em Oftalmologia pela Universidade Federal de Minas Gerais (UFMG). Belo Horizonte (MG). Professor Adjunto de Anatomia da Universidade Federal do Paraná (UFPR). Curitiba (PR).

Médico do Centro Oftalmológico de Curitiba. Curitiba (PR)

Endereço para correspondência: Rogil José de Almeida Torres - Praça Ruy Barbosa 827 - Conj 305 Curitiba (PR) CEP 80010-30

E-mail: rjat@terra.com.br

Recebido para publicação em 28.05.2004

Versão revisada recebida em 03.01.2005

Aprovação em 23.03.2005

\section{INTRODUÇÃOO}

A presença de óleo de silicone em olho afácico e sem o diafragma iriano pode provocar complicações como opacificação de córnea e glaucoma refratário. A simples remoção do óleo de silicone resolveria tais problemas na maioria dos casos. Por outro lado, a retirada do óleo de silicone poderia ocasionar, em casos graves, a recorrência do descolamento de retina e conseqüente perda da função visual. Nestes casos, a fixação de prótese de íris tem demonstrado ser um ótimo artifício para a preservação da córnea ${ }^{(1-2)}$. A prótese de íris substitui, com suas limitações, a barreira mecânica iridocristaliniana, entre o segmento anterior e posterior do olho, mantendo o óleo de silicone na câmara vítrea e impedindo a migração para a câmara anterior. Há relatos do uso da prótese de íris na aniridia congênita e traumática para a resolução da fotofobia, "glare", ou baixa da acuidade visual $^{(3-9)}$. Não foi encontrado relato na literatura sobre a utilização da prótese de íris para controlar o glaucoma refratário provocado pela presença de óleo de silicone na câmara anterior.
\end{abstract}




\section{OBJETIVOS}

10 - Descrever o tipo de prótese de íris utilizada e a técnica cirúrgica adotada para o seu implante. $2^{\underline{0}}$ - Discutir as opções de tratamento para a correção do glaucoma refratário, provocado pela presença de óleo de silicone na câmara anterior, demonstrando assim, a eficácia do procedimento adotado neste caso.

\section{MÉTODOS}

Paciente do sexo masculino, 24 anos de idade, sofreu trauma no olho esquerdo por estilhaços de projétil de arma de fogo no dia 15 de março de 1999. Ferimento palpebral, lesão corneana, aniridia parcial, catarata traumática, hemorragia vítrea, diálise, ruptura e descolamento de retina foram as lesões ocasionadas por dois fragmentos metálicos que penetraram e se alojaram no interior do globo ocular. O paciente foi submetido à sutura palpebral, sutura corneana e remoção do cristalino em outro serviço e apresentava acuidade visual de percepção luminosa. No dia 18 de março de 1999, foi realizada vitrectomia posterior via pars plana, remoção de corpos estranhos metálicos intra-oculares, endofotocoagulação ao redor das soluções de continuidade retinianas, introflexão escleral $360^{\circ} \mathrm{com}$ pneu de silicone número 287 e injeção intra-ocular de óleo de silicone, 5.000 centistokes. No pós-operatório imediato, ao exame de biomicroscopia, apresentava óleo de silicone na câmara anterior. No dia 6 de maio de 1999 apresentava contagem de células endoteliais de 1.317 células $/ \mathrm{mm}^{2}$, pressão intra-ocular de $16 \mathrm{mmHg}$ e óleo de silicone emulsificado e não emulsificado na câmara anterior (Figura 1). A contagem endotelial estabilizou, porém a pressão intra-ocular aumentou de forma gradativa. No dia 16 de julho de 1999 a pressão intraocular era de $25 \mathrm{mmHg}$, a despeito de usar inibidor da anidrase carbônica $250 \mathrm{mg}$ via oral de 6 em 6 horas, maleato de timolol colírio $0,5 \%$ de 12 em 12 horas e alfa-agonista colírio de 12 em 12 horas. A câmara anterior ampla e preenchida, quase que completamente, pelo óleo de silicone. À gonioscopia revelou estruturas do ângulo até o esporão escleral e pigmentação de ++ (moderada) no trabéculo. No dia 18 de agosto de 1999 foi realizada a fixação transescleral da prótese de íris.

Descrição da prótese de íris (figura 2):

1. Material: PMMA

2. Diâmetro total da prótese (de háptico a háptico): $12,5 \mathrm{~mm}$

3. Diâmetro da lente: $10 \mathrm{~mm}$

4. Diâmetro da área óptica: $5 \mathrm{~mm}$

5. Orifícios nos hápticos para fixação transescleral

Descrição da técnica cirúrgica (Figura 3):

1. Injeção peribulbar com bulpivacaína 0,75

2. Assepsia com iodopovidona

3. Colocação de campos descartáveis

4. Colocação de blefarostato

5. Peritomia conjuntival, em região limbar, nos quadrantes superiores

6. Ampla exposição escleral

7. Cauterização dos vasos episclerais

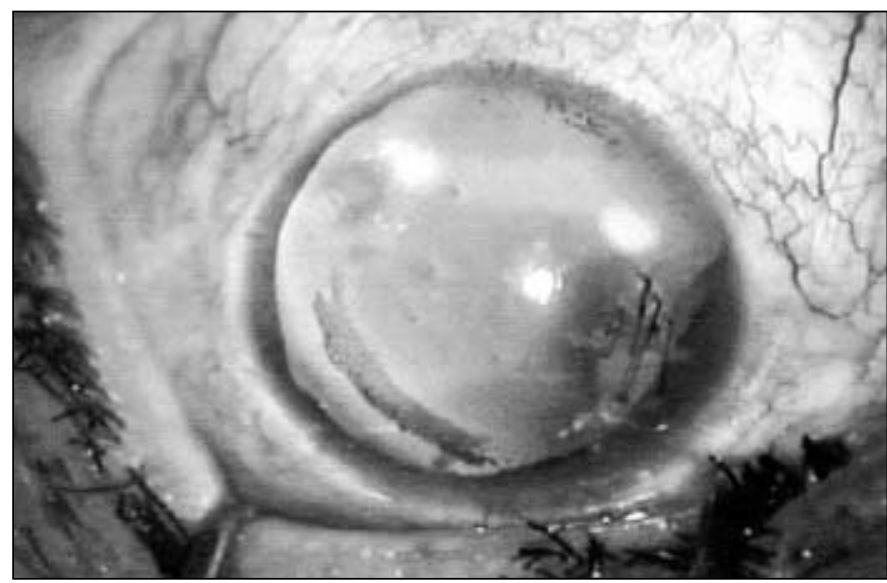

Figura 1 - Sutura corneana. Óleo de silicone emulsificado na câmara anterior. Aniridia parcial

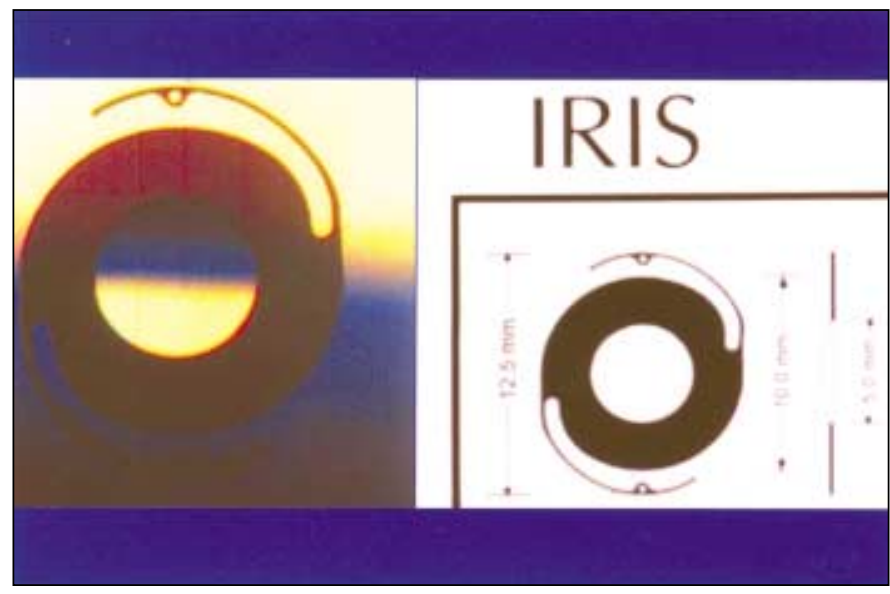

Figura 2 - Prótese de íris fabricada pela Mediphacos ${ }^{\circledR}$. Similar à lente intra-ocular de Morcher 67G

8. Realização de retalhos esclerais triangulares, de base limbar, às 3 e 9 horas, para sepultar posteriormente o fio de prolene

9. Transfixação com fio de prolene 10-0, no leito escleral, a $1 \mathrm{~mm}$ do limbo, das 3 às 9 horas $\mathrm{O}$ fio de prolene apresentava agulha reta numa extremidade e curva em outra

10. Incisão tunelizada da esclera a aproximadamente $1 \mathrm{~mm}$ do limbo e de $11 \mathrm{~mm}$ de extensão

11. Exteriorização do fio de prolene seguida de sua secção.

12. Fixação de cada extremidade do fio de prolene aos respectivos orifícios dos hápticos da lente

13. Preenchimento da câmera anterior com viscoelástico

14. Introdução da prótese de forma lenta na câmara anterior, com ajuste através da tração dos fios de prolene

15. Após o posicionamento da prótese na câmara anterior, foi realizada a sutura do fio de prolene nos sulcos esclerais de 3 e 9 horas

16. Realizou-se a sutura da incisão tunelizada com fio mononylon $10-0$, com pontos em " $X$ " 


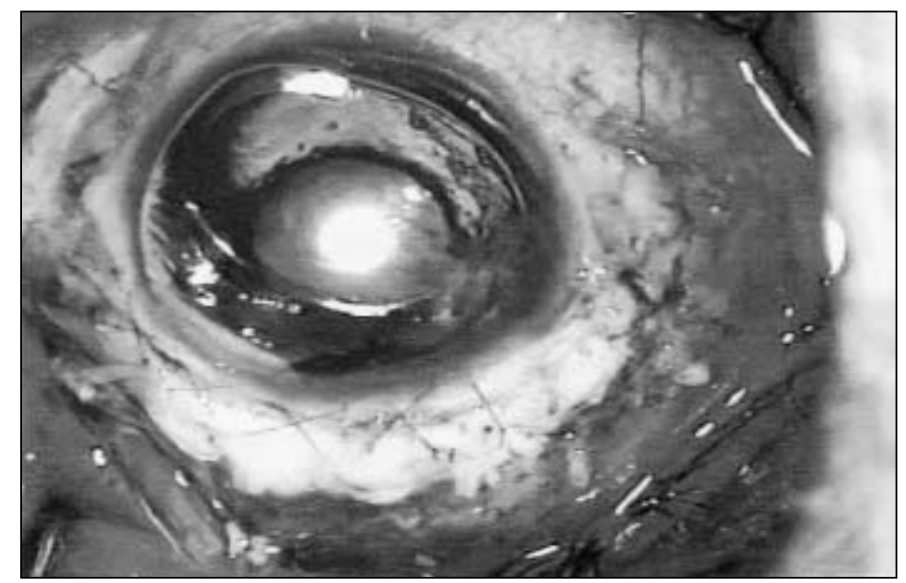

Figura 3 - Fixação transescleral da próteses de íris

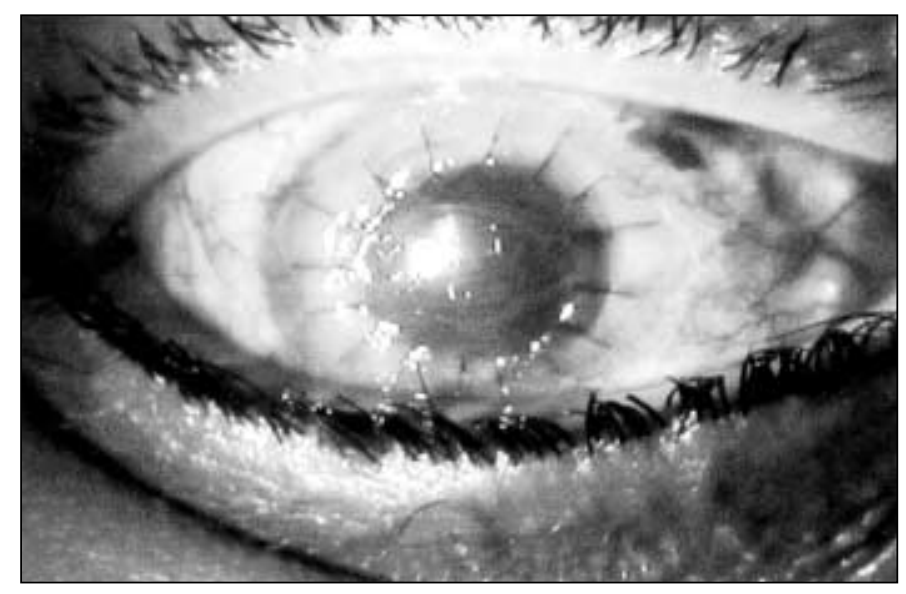

Figura 4 - Pós-operatório do transplante de córnea

17. Aspiração do viscoelástico presente na câmara anterior 18. Sutura dos retalhos esclerais de 3 e 9 horas, com vicryll 8-0

19. Sutura conjuntival com fio vicryll 8-0

20. Injeção subconjuntival de antibiótico associada a corticóide

O paciente foi avaliado no pós-operatório através da acuidade visual, biomicroscopia, pressão intra-ocular com tonômetro de aplanação de Goldmann (Haag-Streit 900), contagem de células endoteliais e gonioscopia. Prescrito colírio de tobramicina associado a dexametasona, 5 vezes ao dia, durante 1 mês. Realizadas avaliações diárias nos primeiros 15 dias, uma vez por semana durante 1 mês, no 3 - mês, $6^{\circ}$ mês e a cada 4 meses até o dia 19 de maio de 2003. Tempo de acompanhamento de aproximadamente 45 meses.

\section{RESULTADOS}

Após a cirurgia de fixação transescleral da prótese de íris, apresentou acuidade visual de conta dedos a 2 metros. À biomicroscopia observou-se centralização da prótese de íris, células +/IV e presença de pequena bolha de óleo de silicone na câmara anterior, que foi removida posteriormente através de paracentese. A pressão intra-ocular de $10 \mathrm{mmHg}$, sem uso de qualquer medicamento hipotensor ocular. Contagem de células endoteliais de 970 células $/ \mathrm{mm}^{2}$. À gonioscopia, verificou-se a alça da prótese no sulco ciliar e algumas goniosinéquias nos locais de íris remanescente.

Posteriormente ocorreu redução gradativa do número de células endoteliais ocasionando opacificação da córnea. $\mathrm{O}$ exame de oftalmoscopia binocular indireta demonstrou pregueamento da retina ao redor da ruptura posterior e tração da retina periférica inferior. No dia 13 de maio de 2002 foi submetido a transplante de córnea (Figura 4). A visão estabilizou em conta dedos a 1 metro e a pressão intra-ocular em $14 \mathrm{mmHg}$, com uso tópico de alfa-agonista. No dia 19 de maio de 2003, após 45 meses da cirurgia do implante da prótese, o quadro encontrava-se inalterado.

\section{DISCUSSÃO}

Antes de tomarmos a decisão para o implante da prótese de íris, ponderamos sobre as várias condutas que poderiam ser pensadas para a resolução da hipertensão ocular, da preservação da córnea e da função retiniana. Entre elas destacamos:

$1^{\text {a) }}$ Iridectomia inferior proposta por $\mathrm{Ando}^{(10)}$, que permite o fluxo de aquoso para a câmera anterior, prevenindo assim o glaucoma por bloqueio pupilar. Este procedimento não foi realizado devido à ausência parcial do tecido iriano.

$2^{2}$-) A simples remoção do óleo de silicone conduziria, com grande probabilidade, ao redescolamento de retina e conseqüente perda da função visual.

3a) A troca de óleo de silicone não foi realizada porque, obviamente, o novo produto também migraria para a câmera anterior.

4a ) A cirurgia fistulante não foi realizada devido à fibrose conjuntival além de que, esta fístula seria obstruída pelo óleo.

5a) A hipótese de colocação do implante valvular foi descartada pela provável obstrução que o óleo provocaria, devido à alta densidade, ou pela possível perda do óleo pelo tubo.

6a) A destruição térmica do corpo ciliar, ciclodestruição por laser diodo ou por crioterapia, provavelmente reduziria a pressão intra-ocular, porém não impediria a lesão do endotélio corneano pelo óleo de silicone.

Atribuímos a hipertensão ocular à presença do óleo de silicone na câmara anterior, interferindo mecanicamente no escoamento do humor aquoso pela malha trabecular. Sendo assim, a única alternativa que encontramos para remover o óleo de silicone da câmara anterior, sem retirá-lo da câmara vítrea, foi o implante da prótese de íris.

Ao optarmos pelo implante, ponderamos sobre as complicações que o procedimento poderia induzir: Hemorragia expulsiva $^{(4)}$, por haver necessidade de ampla abertura de câmara anterior para a introdução da prótese, em olho já traumatizado e hipertenso, descompensação endotelial e hipertensão ocular crônica ${ }^{(5,7-8)}$.

O procedimento cirúrgico ocorreu sem intercorrências per- 
operatórias. A técnica cirúrgica adotada assemelha-se à descrita na literatura ${ }^{(11)}$. Ao longo de 2 anos observamos diminuição gradativa do número de células endoteliais e conseqüente opacificação corneana. A descompensação endotelial poderia ser atribuída a alguns fatores, entre eles destacamos:

- Tipo de prótese de íris utilizada. Verificamos na literatura, próteses com chanfradura inferior, simulando a iridectomia inferior proposta por Ando, associada a orifício central ${ }^{(1)}$ ou a amplas aberturas laterais ${ }^{(2)}$. O objetivo da abertura pupilar ou das amplas aberturas laterais é de permitir melhor circulação de aquoso para a câmara anterior, preservando assim o endotélio corneano $^{(1)}$. Por outro lado, foram descritos vários relatos utilizando o mesmo tipo de prótese que empregamos neste caso, ou seja, sem chanfradura inferior, abertura central ou aberturas laterais e com índice de sucesso considerado bom ${ }^{(3-6,9)}$.

- Reação inflamatória crônica ${ }^{(5-8)}$.

- Aumento da pressão intra-ocular ${ }^{(12)}$.

- Possível dano endotelial cumulativo ocasionado pelas múltiplas cirurgias ${ }^{(13)}$. É importante ressaltar que o número de células endoteliais encontrava-se reduzido (aproximadamente 1.300 células $/ \mathrm{mm}^{2}$ ) antes do implante da prótese. Considera-se o limite para descompensação da córnea de 400 a 700 células $/ \mathrm{mm}^{2(14)}$.

Após aproximadamente 33 meses da cirurgia da fixação transescleral da prótese de íris, o paciente foi submetido à cirurgia de transplante de córnea. Com 12 meses de evolução, observamos manutenção da transparência corneana, a despeito da manutenção do óleo de silicone intra-ocular, na câmara vítrea, e da mesma prótese de íris.

A respeito do aumento da pressão intra-ocular, observado após a cirurgia de transplante de córnea, podemos vinculá-lo à atividade inflamatória crônica da câmara anterior ${ }^{(15)}$, ao acometimento traumático da malha trabecular ${ }^{(5)}$, à introflexão escleral, à obstrução mecânica da malha trabecular por gotículas de óleo de silicone ${ }^{(16)}$, ao uso tópico de anti-inflamatório hormonal ${ }^{(17)}$ ou à associação de todos estes fatores. Esta hipertensão foi tratada com êxito com colírio alfa-agonista.

A despeito das complicações que possam ocorrer com a cirurgia de fixação transescleral da prótese de íris, esta é ainda a única opção que temos quando coexistem fatores críticos como: ausência do diafragma iriano, afacia e impossibilidade da remoção do óleo de silicone, devido a inexorável recorrência de descolamento de retina. Esta conduta tem potencial para controlar a pressão intra-ocular, se o óleo de silicone estiver obstruindo a malha trabecular, e/ou preservar a transparência corneana, impedindo o contato do óleo de silicone com o endotélio corneano. Talvez, no futuro, possam ser pesquisados produtos sintéticos ou biológicos similares anatomicamente ao diafragma irido-cristaliniano, que impeçam a migração do óleo de silicone para a câmara anterior e permitam melhor circulação do aquoso.

\section{ABSTRACT}

The objective of this report is to demonstrate the effectiveness of an iris prosthesis to treat a refractory glaucoma induced by silicone oil in the anterior chamber. This case is about a patient who suffered a trauma caused by firearm shrapnel. A vitreous-retinal surgery was performed to remove intraocular foreign matter and to realign the retina that was detached. Due to the partial traumatic aniridia, silicone oil that was introduced in the vitreous chamber to keep the retina in place migrated to the anterior chamber, resulting in the decrease of endothelium cells and uncontrollable intraocular pressure. We performed transscleral fixation of the iris prosthesis to correct these problems. After a 45-month period of evolution, sight became stable at the 1 meter finger-count distance and intraocular pressure at $14 \mathrm{mmHg}$ We may conclude that the triad that consists of lack of: iris diaphragm, aphakia and silicone oil that could not be removed because of inexorable occurrence of detachment of the retina should lead the surgeon to consider transscleral fixation of the iris prosthesis. This procedure might control intraocular pressure and/or preserve corneal transparency, preventing silicone oil from contact with the trabecular net and the corneal endothelium.

Keywords: Silicone oils/adverse effects; Aniridia; Iris diseases/surgery; Anterior chamber/surgery; Prosthesis implantation; Glaucoma; Case report

\section{REFERÊNCIAS}

1. Heimann K, Konen W. [Artificial iris diaphragm in silicone oil surgery]. Fortschr Ophthalmol. 1990;87(4):329-30. Germany.

2. Moreira Jr. CA, Moreira H, Moreira ATR. Prótese de íris para olhos com aniridia e óleo de silicone. Arq Bras Oftalmol. 1996;59(2):186-90.

3. Burk SE, Da Mata AP, Snyder ME, Cionni RJ, Cohen JS, Osher RH. Prosthetic iris implantation for congenital, traumatic, or functional iris deficiencies. J Cataract Refract Surg. 2001;27(11):1732-40.

4. Dong X, Yu B, Xie L. Black diaphragm intraocular lens implantation in aphakic eyes with traumatic aniridia and previous pars plana vitrectomy. J Cataract Refract Surg. 2003;29(11):2168-73.

5. Thompson C G, Fawzy K, Bryce IG, Noble BA. Implantation of a black diaphragm intraocular lens for traumatic aniridia. J Cataract Refract Surg. 1999; 25(6):808-13.

6. Omulecki W, Synder A. Pars plana vitrectomy and transscleral fixation of black diaphragm intraocular lens for the management of traumatic aniridia. Ophthalmic Surg Lasers. 2002;33(5):357-61.

7. Sundmacher R, Reinhard T, Althaus C. Black diaphragm intraocular lens for correction of aniridia. Ophthalmic Surg. 1994;25(3):180-5.

8. Sundmacher R, Reinhard T; Althaus C. [Black diaphragm intraocular lens in congenital aniridia]. Ger J Ophthalmol. 1994;3(4-5):197-201. Germany.

9. Beltrame G, Salvetat ML, Chizzolini M, Driussi GB, Busatto P, Di Giorgio $\mathrm{G}$, et al. Implantation of a black diaphragm intraocular lens in ten cases of post traumatic aniridia. Eur J Ophthalmol. 2003;13(1):62-8.

10. Ando F. Intraocular hypertension resulting from pupilary block by silicon oil. Am J Ophthalmol. 1985;99(1):87-8.

11. Freitas JAH, Freitas MML, Mais F, Martinelli A, Gallo A, Martinelli G, et al. Implante secundário de câmara posterior sem cápsula. Arq Bras Oftalmol. 1998; 47(3):143-6.

12. Setãlá K. Corneal endothelial cell density after an attack of acute glaucoma. Acta Ophthalmol (Copenh). 1979;57(6):1004-13.

13. Abrams GW, Azen SP, Barr CC, Lai MY, Hutton WL, Trese MT, et al. The incidence of corneal abnormalities in the silicone study.Arch Ophthalmol 1995; 113(6):764-9.

14. Abib F.C. Pós-operatório e acompanhamento de implante secundário de LIO de câmara posterior. Microscopia especular de córnea; Curitiba: Revinter-RioMed; 1999. p.109-10.

15. Reinhard T, Engelhardt S, Sundmacher R. Black diaphragm aniridia intraocular lens for congenital aniridia: long-term follow-up. J Cataract Refract Surg. 2000; 26(3):375-81.

16. Gao R, Neubauer L, Tang S, Kampik A. [Silicone oil in the anterior chamber]. Graefe's Arch Clin. Exp Ophthalmol. 1989;227(2):106-9. Germany.

17. Goldmann, H. Cortisone glaucoma. Arch Ophthalmol. 1962;68:621-6. 